\title{
Patient communication, confidentiality and consent: radiology policy and practice in Europe. A survey by the European Society of Radiology
}

\author{
European Society of Radiology (ESR)
}

Received: 12 February 2013 / Accepted: 13 February 2013 / Published online: 9 March 2013

(C) The Author(s) 2013. This article is published with open access at Springerlink.com

\begin{abstract}
The data collected in this article were gathered from various practising radiology departments around $\mathrm{Eu}-$ rope. The focus is communication and confidentiality between patient and radiologist, specifically the ramifications of PACS and other electronic systems for the confidentiality of patient information. The differences and similarities in practice across Europe are explored.

Main Messages

- Monitoring of access to confidential patient imaging information does not appear to have kept pace with electronic image storage systems

- Europe lacks a unified approach to define clear guidelines for patient confidentiality and guidance on what constitutes invasion of patient privacy

- Specific training in communication is not routinely incorporated into radiology training schemes
\end{abstract}

Keywords Communication · Confidentiality · Consent · Radiology

\section{Introduction}

Imaging and image-guided therapy are now pivotal to patient management. What the patient is told about the tests or procedures they undergo, how results are communicated to them, and the patient's rights in terms of access to their images and reports has the potential to vary considerably across Europe. The safeguarding and protection of medical information about the patient and their right to confidentiality is also more difficult to maintain in an electronic age of

European Society of Radiology (ESR) $(\bowtie)$

Neutorgasse 9/2a,

1010 Vienna, Austria

e-mail: communications@myESR.org
PACS systems, which store large volumes of data, but are also accessible to a large number of people who have no medical responsibility for the patient.

\section{Questionnaire}

The Audit and Standards subcommittee of the ESR carried out a survey to evaluate practice in Europe and to look for areas of common practice, and areas where practice differs. The questionnaire was sent to National Societies.

\section{Results}

The response rate was $80 \%$ and the results are tabulated below.

1. Do patients receive a written copy of or have electronic access to their radiology reports?

Routinely receive reports or

can access them electronically

$45.5 \%$

Sometimes

$21.2 \%$

Rarely

$24.2 \%$

Never

$9.1 \%$

2. Do patients have a legal right to see their reports if they wish?

Yes

$100.0 \%$

No

$0.0 \%$

3. Do radiologists discuss the imaging findings (particularly ultrasound) directly with the patient

Routinely

$45.5 \%$

Sometimes

$42.4 \%$

Rarely

$12.1 \%$ 
4. Do radiologists receive training on communication, such as giving bad news to patients?

Now routine, as part of training

$15.2 \%$

Sometimes

$21.2 \%$

Rarely, depends on individual

initiative of radiologist

$45.5 \%$

Never

$18.2 \%$

5. Is there a national policy or guidance to doctors/ radiologists on disclosing patient information to anyone not directly involved in their care?

Yes

$75.8 \%$

No

$24.2 \%$

6. Is there a mechanism for doctors to be in some way disciplined or punished if they misuse patient information or breach patient consent?

Yes

$84.8 \%$

No

$15.2 \%$

7. Are there any National Society or other guidelines on who should/has the right to view An individual patient's films or images and reports?

Yes

$63.6 \%$

No

$18.2 \%$

Not aware of any

$18.2 \%$

8. Is there any monitoring of who has looked at individual patient reports/images?

Yes, these data are routinely kept for review

$45.5 \%$

Not usually

$36.4 \%$

Not aware of any

$18.2 \%$

9. Do patients have to give consent for their images to be published?

Yes, specific written consent

$18.8 \%$

Yes, oral consent

$3.1 \%$

Variable practice

$15.6 \%$

No, if anonymised images are used

$62.5 \%$

10. Do patients have to give consent for their images to be used for teaching?

Yes, specific written consent

$6.3 \%$

Yes, oral consent

$3.1 \%$

Variable practice (e.g. local rules

relating to the institution)

$40.6 \%$

No, consent is assumed

$50.0 \%$

11. Do patient give written consent for interventional radiology procedures?

Yes

$71.9 \%$

No

$12.5 \%$

Sometimes
12. Is written consent given for all procedures or investigations involving ionising radiation?

Yes

$28.1 \%$

No

$71.9 \%$

13. Do patients routinely give written consent for contrast to be administered?

Yes

$43.8 \%$

No

$56.3 \%$

14. Are patients given written information about radiation risks for all investigations involving radiation?

$\begin{array}{ll}\text { Yes } & 21.9 \% \\ \text { No } & 37.5 \% \\ \text { Sometimes } & 40.6 \%\end{array}$

\section{Discussion}

Some areas of broad agreement and common practice and other areas where there was significant variation in practice emerged.

\section{Communication}

All respondents agreed that patients had a legal right to see their radiology reports if they wished/requested, but the survey indicated that routine practice varies across Europe. Slightly under half of respondents indicated that patients routinely received reports and approximately one quarter that patients rarely received them. Nine per cent of respondents replied that patients never receive written reports. Free text comments indicated that practice may vary across individual healthcare systems, with differences between private and state run services, and inpatient or outpatient referrals. It is clear that although the legal right exists, in practice this may be expensive to implement routinely and difficult to achieve. There is also the perceived risk that patients could be unnecessarily alarmed by radiology reports that need to be explained in person and in the context of their illness.

With respect to direct discussion of the imaging findings between patients and radiologists when the patient asked, results showed that in under half of the participating countries the radiologist would routinely discuss the imaging findings with the patient, and in $12 \%$ this rarely occurred. Free text comments indicated that again there may be variation between private and state-provided care. Other factors cited were the time available and variation depending on the modality, for example more discussion was likely to take place about ultrasound examinations that MRI. One respondent also indicated that radiologists did not discuss the findings because the referring doctors preferred to discuss 
the examination results with the patients (with which the radiologist respondent indicated that they did not agree).

In response to the question of training for radiologists in communication skills such as giving bad news, in only $15 \%$ of cases was this provided as part of radiology training, and just under half of survey respondents indicated there was rarely any training provided and it would depend on the individual initiative of each radiologist.

The survey results highlighted several issues related to communication. As radiology moves further towards openness and the provision of reports to patients, greater direct communication between radiologists and patients may follow [1]. This may make the role of the radiologist clearer to patients, but direct discussions or even electronic communication between radiologists and patients is potentially very time-consuming, with the potential to reduce radiologists' reporting productivity. Direct communication is also difficult to achieve with teleradiology. The survey also indicated that training in communication skills is not widely incorporated into radiologists' training to prepare them for situations such as having to give bad news to patients.

\section{Confidentiality}

Patients have a right to expect that their own privacy will be respected and that only those who need to know about their health should access their records [3, 4].

Three quarters of national societies indicated that there was a national policy on disclosing patient information, but less than two thirds had professional guidelines on who had the right/should view patients' images or reports. Most countries had regulations in place so that a doctor could be disciplined or punished if they misused patient information, but in $15 \%$ of cases no such regulation is in place. In the era of electronic access to records, password protection can be misused, and large numbers of people may have access to an imaging archive, which may be a temptation to view others' imaging information just for personal interest. Proving wrongful access to patient information is however difficult as over half of national societies responded that there is no monitoring of who has accessed individual patients' images or reports.

One respondent cited a judgement from the European Court, I vs. Finland. The applicant 'I' stated that her private medical records were accessed by other people (as a result of which she possibly lost her job as a nurse). The access was not recorded, as there were no records of this at the time (around 1992). The court decided that the hospital was controlled by the State, and as such Finland was responsible for the actions there. The court also stated that personal information relating to a patient undoubtedly belongs to his or her private life. Therefore Article 8, freedom to a private life, is applicable in this case. The European Court of Human Rights found that a person's right to respect of their private life (under the ECHR) may be breached where the State fails to take appropriate steps to secure data, so that it cannot be accessed improperly [2].

Confidentiality of imaging records may be an area where policy has not kept up with technological advances. When patient records were in the form of film and paper reports, access by any individual was usually obvious. With electronic storage anyone with access to a password can browse a whole archive. It is apparent from the survey that monitoring of this browsing is not usually routine, and there is at least the potential for misuse.

\section{Consent}

Consent is difficult to define as it can be given verbally or in writing, but in both cases true informed consent can only be given when the patient fully understands the procedure and its potential benefits and risks to them as an individual. The benefits to the doctor of written consent is the presence of a permanent record of patient agreement, and differences in policy for obtaining written consent was apparent from the survey. In the case of interventional procedures, written consent was obtained routinely in $72 \%$ of cases. Exactly the reverse is seen for written consent for examinations using ionising radiation, where $72 \%$ did not involve obtaining written consent. In the middle is the rate of written consent for contrast administration, which was split $44 / 56 \%$ yes/no.

From the survey it appears that the likelihood of written consent being obtained is related to the perceived immediate risk to the patient. The legal status of written consent however may not be the same throughout Europe. In some countries it may be a medico-legal requirement; in others the presence of a written consent document is not deemed to prove that the patient has been adequately informed and has provided informed consent to undergo the investigation or procedure. For the use of images in teaching and research, it seems that written consent is not often obtained, being $6 \%$ and $19 \%$ respectively. For teaching, consent is assumed, but not obtained in $50 \%$ of cases in accordance with local policy, and for publication written consent is not obtained in the majority of cases if anonymised images are used.

\section{Conclusions}

The survey revealed both similarities in practice and differences. It highlights how policy in respect to confidentially may not have kept up with changes in technology. Also, as patients become better informed, they may require more explanation and information about the risks and benefits of the procedures that they undergo in radiology departments. Similarly, with more openness and direct patient access to their records, radiologists may have an increasing need to communicate directly with patients, which is not usually 
part of their training, and may be difficult to achieve in an era of teleradiology.

Acknowledgments This paper was kindly prepared by the ESR Subcommittee on Audit \& Standards (Chairperson: E. Jane Adam. National Societies Committee Chairman: Luis Donoso. Members: Maurizio Centonze, Elisabeth Dion, Birgit Ertl-Wagner, Roberts Manns, Peeter Ross, Hatem Alkadhi, Sofia Gourtsoyianni) on behalf of the European Society of Radiology (ESR). It was approved by the ESR Executive Council in January 2013.

Open Access This article is distributed under the terms of the Creative Commons Attribution License which permits any use, distribution, and reproduction in any medium, provided the original author(s) and the source are credited.

\section{References}

1. Berlin L (2007) Communicating results of all radiologic examinations directly to patients: has the time come? AJR Am J Roentgenol 189:1275-1282

2. European Court of Human Rights (ECHR) article 8

3. Directive 2002/58/EC of the European Parliament and of the Council of 12 July 2002 concerning the processing of personal data and the protection of privacy in the electronic communications sector (Directive on privacy and electronic communications)

4. Directive 95/46/EC of the European Parliament and of the Council of 24 October 1995 on the protection of individuals with regard to the processing of personal data and on the free movement of such data 